O Open Access Full Text Article

HYPOTHESIS

\title{
Frontal Fibrosing Alopecia. An Example of Disrupted Aryl Hydrocarbon Receptor-Mediated Immunological Homeostasis in the Skin?
}

This article was published in the following Dove Press journal:

Clinical, Cosmetic and Investigational Dermatology

\section{Rowland Noakes}

Queensland Institute of Dermatology, South Brisbane, Queensland, Australia
Correspondence: Rowland Noakes Queensland Institute of Dermatology, 10 Browning Street, South Brisbane, Queensland, Australia

Tel +6I 733294400

Fax +6I 733294455

Email ky_n_urenine@hotmail.com.au

\begin{abstract}
Sun protection has been recommended by public health authorities to reduce the risk of keratinocyte and melanocyte tumors, yet some sun exposure is required for vitamin D synthesis. Frontal fibrosing alopecia (FFA) is a cicatricial alopecia that has been reported to have an association with facial photoprotection. The brief review proposes the hypothesis that FFA arises as a result of excessive facial photo-protection with a resultant disturbance in immunological homeostasis mediated via the aryl hydrocarbon receptor-kynurenine pathway axis (AHR/KP) leading to the collapse of immune privilege at the hair bulge.
\end{abstract}

Keywords: sun exposure, aryl hydrocarbon receptor, frontal fibrosing alopecia, 6-formylindolo [3, 2-b]carbazole

\section{Introduction}

Frontal fibrosing alopecia is a cicatricial alopecia seen most commonly in postmenopausal women which involves the anterior hairline and eyebrows. First described by Kossard, ${ }^{1}$ it is considered a variant of lichen planopilaris. The incidence is reported to be rising suggesting a role for environmental factors. Recent attention has focused on the role played by sunscreens and facial care products $^{2}$ and this was supported in a recent multivariate analysis. ${ }^{3}$ More recently the role of sun exposure in immunological homeostasis has been explored. ${ }^{4}$

The aryl hydrocarbon receptor (AHR) is a cytosolic receptor for low molecular weight molecules, the most widely recognized ligand being 2,3,7,8-tetrachlorodibenzo- $p$-dioxin (TCDD), which plays an important role in immunological homeostasis in the skin. ${ }^{5} 6$-formylindolo[3,2-b] carbazole (FICZ) is a potent endogenous ligand of the AHR produced by photo-oxidation of the essential amino acid L tryptophan which displays pro-inflammatory properties at lower concentrations and anti-inflammatory properties at higher concentrations. ${ }^{6}$

The hair follicle is a cutaneous appendage that displays immunological privilege, ${ }^{7}$ collapse of which results in TH1 mediated immune destruction of the epithelial hair follicle stem cells (eHFSC) ${ }^{8}$ and permanent hair loss. This article will review our current understanding of the pathogenesis of frontal fibrosing alopecia and the potential role the AHR may play. It postulates that a certain level of sun exposure is required to maintain immunological homeostasis within the skin and frontal fibrosing alopecia arises due to a disruption immunological homeostasis mediated via the AHR/KP axis. 


\section{Discussion}

\section{The Pathogenesis of Frontal Fibrosing Alopecia}

The hair follicle is a site of immunological privilege. Several mechanisms are at play in the maintenance of immunological privilege.

1. There is reduced or absent expression of the major histocompatibility complex 1 (MHC 1) beta 2 microglobulin complex rendering expression of endogenous peptides ineffectual or impossible. ${ }^{9}$

2. There is a downregulation of MHC class 2 in antigen-presenting cells (APC). ${ }^{9}$ This has been reported to be mediated by AHR agonists. ${ }^{10}$

3. A local immunoinhibitory medium is established by the secretion of immunoinhibitory molecules. These include alpha melanocyte-stimulating hormone $(\alpha-\mathrm{MSH})$, transforming growth factor-beta 1 and 2 (TGF- $\beta 1,2$ ), interleukin 10 (IL-10), cortisol and enhanced indoleamine 2,3 di-oxygenase expression (IDO) which depletes the micro-environment of the essential amino acid $\mathrm{L}$ tryptophan limiting $\mathrm{T}$ cell proliferation. ${ }^{11}$ A breach of immune privilege leads to TH1-mediated destruction of the eHFSC and permanent destruction of the hair follicle.

4. Expression of peroxisome proliferator-activated receptor-gamma (PPAR- $\gamma$ ) is down-regulated in lichen planopilaris. ${ }^{12}$

5. CD200 is a membrane glycoprotein highly expressed in human follicular stem cells which represents a "no danger signal" to immunosurveillance systems. ${ }^{13}$ Interaction with the CD200 receptor (CD200R) attenuates inflammatory responses and protects eHFSC. CD200 expression has been reported to be a factor in the maintenance of hair follicle immune privilege. ${ }^{13}$

A loss of immunological privilege leads to a CD8 mediated immune destruction, Epithelial-mesenchymal transition (EMT) is involved in the loss of follicles. ${ }^{14}$ TGF- $\beta 1$, epidermal growth factor (EGF) and interferongamma (IFN- $\lambda$ ) are involved in this process. ${ }^{15}$

\section{The Aryl Hydrocarbon Receptor}

The aryl hydrocarbon receptor (AHR) is a cellular receptor for low molecular weight molecules which plays an important role in cellular oxidation/antioxidation reactions, response to ultraviolet light, pigmentation, and immune regulation.
It is a cytoplasmic receptor that forms part of a multiprotein complex. Chaperoning proteins include heat shock protein 90 (hsp90), hsp23, and hepatitis $\mathrm{B}$ virus $\mathrm{X}$-associated protein 2 (XAP2). Pp60 ${ }^{\text {src }}$ is an associated signaling partner that is released into the cytosol on ligand binding. On ligand binding, the AHR translocates to the nuclear membrane where it binds to the aryl hydrocarbon receptor nuclear transporter (AHRNT). This promotes the expression of genes containing xenobiotic response elements (XRE) in their promotors. Principal amongst these are the cytochrome P450 1A1, 1A2, 1B1 monooxygenases which introduce functional groups prior to conjugation with water-soluble molecules by the Phase 2 detoxification enzymes.

Phase 2 enzyme regulation is via the nuclear factor erythroid derived 2, like 2 transcription factor (Nrf2) which also contains XRE in its promoter allowing the coordination of Phase 1 and 2 detoxification reactions. Control is provided firstly by the synthesis of the repressor protein, the aryl hydrocarbon receptor repressor (AHRR) and export of the AHR for cytosolic degradation via the $26 \mathrm{~S}$ proteasome pathway.

Cross talk exists with the retinoblastoma protein, the estrogen receptor (ER), the retinoic acid signaling pathway and nuclear factor kappa light chain enhancer of activated $\beta$ cells $(\mathrm{NF}-\mathrm{\kappa B})$ pathway. In addition, Pp60 src binds to the EGF receptor initiating mitogen-activated protein kinase (MAPK) signaling. Thus, the AHR can modify multiple signaling pathways. $^{16}$

\section{AHR Involvement in the Pathogenesis of Frontal Fibrosing Alopecia}

Factors mediating immune privilege at the hair follicle are illustrated in Figure 1.

\section{The KP/AHR Axis}

The KP is the metabolic pathway by which L tryptophan is metabolized to nicotinamide. In addition to providing an endogenous supply of nicotinamide, it serves important immunoregulatory roles. The rate-limiting enzyme is indoleamine 2, 3-dioxygenase (IDO). Although the best recognized inducer of this enzyme is interferon-gamma (IFN- $\lambda$ ), it is also induced by the AHR. In addition, a metabolic intermediate of the KP, kynurenine is an AHR agonist. ${ }^{17}$ As well as depleting the micro-environment of the essential amino acid L tryptophan thereby inhibiting immune proliferation, many of the intermediates display immunoregulatory properties. 


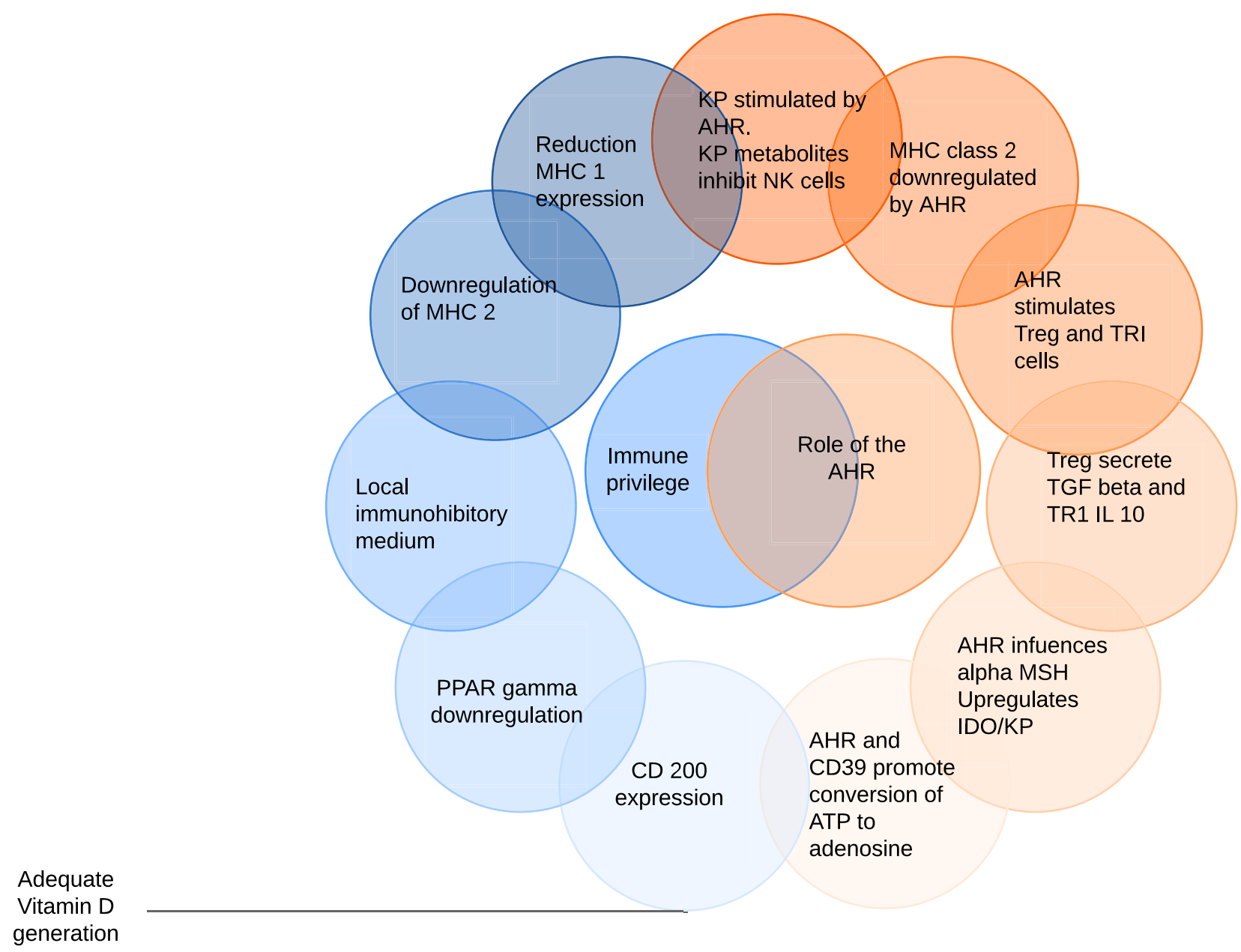

Figure I Factors mediating immune privilege at the hair follicle are illustrated on the left of the diagram with the role of the aryl hydrocarbon receptor (AHR) on the right. Cells which do not express MHC I are normally subject to natural killer (NK) attack. AHR activity stimulates indoleamine 2,3 di-oxygenase (IDO) and thus the kynurenine pathway (KP), metabolites of which inhibit NK activity. MHC class 2 expression is also downregulated by the AHR. In addition, AHR agonists facilitate the development of regulatory T cells (Treg) and IL- 10 secreting TRI cells which secrete TGF $\beta$ and IL- I0. These in combination with $\alpha-M S H$ create a local immunoinhibitory medium. Finally, the AHR promotes the conversion of extracellular ATP to adenosine which has immune-inhibitory activity. CD200 expression requires an adequate supply of vitamin D which is also dependent on sun exposure.

Natural killer (NK) cells are a component of the innate lymphoid system which preferentially target cells that lack the surface expression of MHC class 1 molecules. Inhibition of NK activity is thus required for the maintenance of immune privilege at the follicular unit. KP metabolites that inhibit NK activity include anthranilic acid, 3-hydroxykyurenine, 3-hydroxyanthranilic acid, quinolinic acid and picolinic acid. ${ }^{18}$

\section{Alpha MSH}

Alpha MSH displays immunosuppressive properties in the $\operatorname{skin}^{19}$ and is influenced by AHR activity. ${ }^{20}$

\section{Regulatory T Cells and TGF $\beta$ I,2 and IL-I0}

FoxP3 T regulatory cells (Treg) and IL-10 secreting type 1 regulatory $\mathrm{T}$ cells (TR1) are best recognized regulatory $\mathrm{T}$ cell groups. AHR activation increases Treg cells and is important in the differentiation of TR1 cells. ${ }^{21}$ TGF $\beta$ is utilized by Treg to suppress cytotoxic tumor responses ${ }^{22}$ and latent TGF $\beta$ is carried on the surface of Treg cells linked to the transmembrane protein Glycoprotein A repetitions predominant protein (GARP), via disulfide bonds. ${ }^{23}$ Activation is via $\alpha \mathrm{V}$ class integrins.

IL-10 is secreted by TR1 cells. The AHR interacts with the transcription factor c-Maf to promote the secretion of IL-10 and IL-21. ${ }^{24}$ In addition, the AHR and Stat 3 combine to deplete the extracellular environment of Adenosine Triphosphate (ATP) via CD39 generating immunosuppressive adenosine. ${ }^{25}$

\section{Epithelial-Mesenchymal Transition}

AHR activity has been reported both to promote and to inhibit EMT depending on the system and cell type being 
investigated. As the AHR promotes the differentiation of Treg which promotes a TGF $\beta$ enhanced environment, ${ }^{21}$ an expectation of enhanced EMT would not be unexpected. As expected, however, a balance of factors is at play. In a mouse model, the AHR agonist of relevance FICZ inhibited TGF $\beta$-induced EMT. ${ }^{26}$

Additionally, IFN- $\lambda$ expression, which plays a role in EMT, ${ }^{14}$ is influenced by the AHR. IFN- $\lambda$ signals through the Janus kinases, Jak 1 and 2 activating Stat 1 . Stat 1 is inhibited via the AHR. ${ }^{20}$

\section{Peroxisome Proliferator-activated Receptor Gamma (PPAR- $\gamma$ )}

PPAR- $\gamma$ is one of the three members of the PPAR group with important roles in fatty acid metabolism as well as insulin secretion and sensitivity. Disturbances in PPAR- $\lambda$ are considered to play an essential role in cicatricial alopecia. ${ }^{27}$ The AHR agonist TCDD inhibits PPAR- $\lambda$ mRNA synthesis ${ }^{28}$ yet this is a long-acting and slowly metabolized agonist. The actions of physiological agonists such as FICZ which are rapidly metabolized by the inducible cytochrome P450 system remain to be elucidated and are likely to be far more dynamic.

The AHR is also active at many sites within the follicular unit and the net effect on PPAR- $\lambda$ is likely to be the result of the interplay of several factors. The AHR increases Treg cells ${ }^{20}$ which have been demonstrated to augment the proliferation and differentiation of eHFSC. ${ }^{29}$

\section{CD200/CD200R Axis}

Although there is a paucity of published reports for the role of the AHR in CD200/CD200R expression, CD 200 expression is upregulated by vitamin $\mathrm{D}^{30}$ which is synthesized by the skin on light exposure.

The CD200/CD200R axis however does exert influence by modulation of the KP with CD200 induction of IDO in dendritic cells. ${ }^{31}$

\section{Endocrine Factors}

Frontal fibrosing alopecia is seen predominantly in postmenopausal women. There is cross-talk between the ER and the AHR. Activation of the AHR inhibits estrogen signaling whereas ER- $\alpha$ has a positive role in AHR signaling supportive of the view that AHR signaling decreases in the post-menopausal state. ${ }^{32}$

The 5 alpha-reductase inhibitors finasteride and dutasteride which inhibit the conversion of testosterone to dihydrotestosterone (DHT) have been reported to be efficacious in the management of frontal fibrosing alopecia. Of interest, FICZ has been reported to inhibit the formation of DHT in the prostatic adenocarcinoma cell line $\mathrm{LNCaP}^{33}$

\section{Immunological Attack}

CD8 cells are prominent in the inflammatory infiltrate. ${ }^{8,34}$ AHR activation by TCDD inhibits CD8 function. ${ }^{35}$

\section{AHR Expression}

After binding to the XRE, the AHR is exported to the cytoplasm for degradation by the $26 \mathrm{~S}$ proteasome pathway. After exposure to a ligand, the level of the AHR protein has been found to decrease. ${ }^{36}$ Increased levels of AHR expression have been reported in frontal fibrosing alopecia suggesting that AHR cycling may be reduced. ${ }^{37}$

\section{Conclusion}

A possible association between low levels of photoexposure and a loss of immune tolerance first appeared when the association between vitamin $\mathrm{D}$ and multiple sclerosis was documented. ${ }^{38}$ Although vitamin D does have immunoregulatory properties, ${ }^{39}$ other immunoregulatory molecules are generated on light exposure. In this hypothesis, we propose and review the evidence that FFA arises due to the collapse of immune privilege at the hair bulb because photo-protection provided by many facial care products reduces the synthesis of the tryptophan photoproduct FICZ. Of relevance, FICZ is generated most efficiently by UVB but is also capable of being generated by UVA and visible light. ${ }^{5}$ Low levels of FICZ are immunostimulatory with higher levels being immunosuppressive ${ }^{6}$ which would support the proposition that UV protective facial products which allow the transmission of visible light but block UV light may have immunostimulatory properties.

An association between FFA and exposure to alkylphenolic compounds has also been reported. These are environmental toxins with both an endocrine disruption potential and activity at the $\mathrm{AHR}^{40}$ which have been shown to interfere with PPAR- $\gamma$.

The role of genetic factors was explored in a recent genome-wide association study. ${ }^{41}$ Reported associations were with HLA-B*07:02, Cytochrome P450 1B1 (CYP1B1), the ST3GAL1 gene encoding the galactoside sialyltransferase enzyme and possibly the gene SEMA4B. CYPB1 is an AHR dependent monooxygenase supporting the proposal that the AHR plays a significant role in this disorder. ST3GAL1 is reported to play a role in $\mathrm{T}$ cell homeostasis consistent with the role played by $\mathrm{T}$ cells in 
eHFSC destruction. Class 1 HLA antigens are normally absent or expressed only at lower levels in the hair follicle permissive of immune privilege; however, an HLA association may assume significance in the event of immune privilege collapse, mediating ongoing destruction. These reported genetic associations are consistent with the hypothesis outlined.

\section{Disclosure}

The author reports no conflicts of interest in this work.

\section{References}

1. Kossard S. Postmenopausal frontal fibrosing alopecia. Arch Dermatol. 1994;130(6):770. doi:10.1001/archderm.1994.01690060100013

2. Cranwell W, Sinclair R. Sunscreen and facial skincare products in frontal fibrosing alopecia: a case-control study. $\mathrm{Br} J$ Dermatol. 2019;180(4):943-944. doi:10.1111/bjd.17354

3. Moreno-Arrones O, Saceda-Corralo D, Rodrigues-Barata A, et al. Risk factors associated with frontal fibrosing alopecia: a multicentre case-control study. Clin Exp Dermatol. 2018;44(4):404-410. doi:10.1111/ced.13785

4. Matsushima Y, Mizutani K, Yamaguchi Y, Yamanaka K. Vitamin D is no substitute for the sun. J Allergy Clin Immunol. 2019;143 (3):929-931. doi:10.1016/j.jaci.2019.01.004

5. Stockinger B, Meglio P, Gialitakis M, Duarte J. The aryl hydrocarbon receptor: multitasking in the immune system. Annu Rev Immunol. 2014;32(1):403-432. doi:10.1146/annurev-immunol-032713-120245

6. Rannug A, Rannug U. The tryptophan derivative 6-formylindolo [3,2-b]carbazole, FICZ, a dynamic mediator of endogenous aryl hydrocarbon receptor signaling, balances cell growth and differentiation. Crit Rev Toxicol. 2018;48(7):555-574. doi:10.1080/ 10408444.2018.1493086

7. Paus R, Ito N, Takigawa M, Ito T. The hair follicle and immune privilege. J Invest Dermatol Symp Proc. 2003;8(2):188-194. doi:10.1046/j.1087-0024.2003.00807.x

8. Harries M, Meyer K, Chaudhry I, et al. Lichen planopilaris is characterized by immune privilege collapse of the hair follicle's epithelial stem cell niche. J Pathol. 2013;231(2):236-247. doi:10.1002/path.4233

9. Azzawi S, Penzi L, Senna M. Immune privilege collapse and alopecia development: is stress a factor. Skin Appendage Disord. 2017;4 (4):236-244. doi: $10.1159 / 000485080$

10. Bruhs A, Haarmann-Stemmann T, Frauenstein K, Krutmann J, Schwarz T, Schwarz A. Activation of the arylhydrocarbon receptor causes immunosuppression primarily by modulating dendritic cells. J Invest Dermatol. 2015;135(2):435-444. doi:10.1038/jid.2014.419

11. Kinori M, Kloepper J, Paus R. Can the hair follicle become a model for studying selected aspects of human ocular immune privilege? Invest Ophthalmol Visual Sci. 2011;52(7):4447. doi:10.1167/ iovs.10-7154

12. Harries M, Paus R. Scarring alopecia and the PPAR- $\gamma$ connection. J Invest Dermatol. 2009;129(5):1066-1070. doi:10.1038/jid.2008.425

13. Rosenblum M, Yancey K, Olasz E, Truitt R. CD200, a "no danger" signal for hair follicles. J Dermatol Sci. 2006;41(3):165-174. doi:10.1016/j.jdermsci.2005.11.003

14. Harries M, Jimenez F, Izeta A, et al. Lichen planopilaris and frontal fibrosing alopecia as model epithelial stem cell diseases. Trends Mol Med. 2018;24(5):435-448. doi:10.1016/j.molmed.2018.03.007

15. Harries M, Imanishi H, Paus R. Fibrosis and stem cell epithelial-mesenchymal transition in primary cicatricial alopecias. J Am Acad Dermatol. 2019;80(6):e165-e166. doi:10.1016/j.jaad. 2018.12.055
16. Noakes R. The aryl hydrocarbon receptor: a review of its role in the physiology and pathology of the integument and its relationship to the tryptophan metabolism. Int $J$ Tryptophan Res. 2015;8:IJTR. S19985. doi:10.4137/IJTR.S19985

17. Jaronen M, Quintana F. Immunological relevance of the coevolution of IDO1 and AHR. Front Immunol. 2014;5.

18. Lanser L, Kink P, Egger E, et al. Inflammation-induced tryptophan breakdown is related with anemia, fatigue, and depression in cancer. Front Immunol. 2020. doi:10.3389/fimmu.2020.00249

19. Auriemma M, Brzoska T, Klenner L, et al. $\alpha$-MSH-stimulated tolerogenic dendritic cells induce functional regulatory $\mathrm{T}$ cells and ameliorate ongoing skin inflammation. J Invest Dermatol. 2012;132 (7):1814-1824. doi:10.1038/jid.2012.59

20. Abbas S, Alam S, Singh K, Kumar M, Gupta S, Ansari K. Aryl hydrocarbon receptor activation contributes to benzanthrone-induced hyperpigmentation via modulation of melanogenic signaling pathways. Chem Res Toxicol. 2017;30(2):625-634. doi:10.1021/acs. chemrestox.6b00364

21. Gutiérrez-Vázquez C, Quintana F. Regulation of the immune response by the aryl hydrocarbon receptor. Immunity. 2018;48 (1):19-33. doi:10.1016/j.immuni.2017.12.012

22. Chen M, Pittet M, Gorelik L, et al. Regulatory T cells suppress tumorspecific CD8 T cell cytotoxicity through TGF- $\beta$ signals in vivo. Proc Natl Acad Sci. 2004;102(2):419-424. doi:10.1073/pnas.0408197102

23. Stockis J, Colau D, Coulie P, Lucas S. Membrane protein GARP is a receptor for latent TGF- $\beta$ on the surface of activated human Treg. Eur J Immunol. 2009;39(12):3315-3322. doi:10.1002/eji.200939684

24. Wu H, Quintana F, da Cunha A, et al. In vivo induction of Tr1 cells via mucosal dendritic cells and AHR signaling. PLoS One. 2011;6(8): e23618. doi:10.1371/journal.pone.0023618

25. Takenaka M, Robson S, Quintana F. Regulation of the T cell response by CD39. Trends Immunol. 2016;37(7):427-439. doi:10.1016/j. it.2016.04.009

26. Rico-Leo E, Alvarez-Barrientos A, Fernandez-Salguero P. Dioxin receptor expression inhibits basal and transforming growth factor $\beta$ induced epithelial-to-mesenchymal transition. J Biol Chem. 2013;288 (11):7841-7856. doi:10.1074/jbc.M112.425009

27. Harnchoowong S, Suchonwanit P. PPAR- $\gamma$ agonists and their role in primary cicatricial alopecia. PPAR Res. 2017;2017:1-12. doi:10.11 $55 / 2017 / 2501248$

28. Alexander DL e. Aryl-hydrocarbon receptor is an inhibitory regulator of lipid synthesis and of commitment to adipogenesis. - PubMed NCBI. Ncbi.nlm.nih.gov. Available from: https://www.ncbi.nlm.nih. gov/pubmed/9788873. 2020. Accessed April 25, 2020..

29. Ali N, Zirak B, Rodriguez R, et al. Regulatory $T$ cells in skin facilitate epithelial stem cell differentiation. Cell. 2017;169 (6):1119-1129. doi:10.1016/j.cell.2017.05.002

30. Tiede S, Ramot Y, Bohm K, Plate C, Paus R. Session N: cicatricial alopecia and stem cells. Int J Trichology. 2011;3(S29).

31. Mellor A, Lemos H, Huang L. Indoleamine 2,3-Dioxygenase and tolerance: where are we now? Front Immunol. 2017;8. doi:10.3389/ fimmu.2017.01360.

32. Matthews J, Gustafsson J. Estrogen receptor and aryl hydrocarbon receptor signaling pathways. Nucl Recept Signal. 2006;4(1): nrs.04016. doi: $10.1621 / \mathrm{nrs} .04016$

33. Arabnezhad M, Montazeri-Najafabady N, Chatrabnous N, Ghafarian Bahreman A, Mohammadi-Bardbori A. Anti-androgenic effect of 6-formylindolo[3,2-b]carbazole (FICZ) in LNCaP cells is mediated by the aryl hydrocarbon-androgen receptors cross-talk. Steroids. 2020;153:108508. doi:10.1016/j.steroids.2019.108508

34. Del Duca E, Ruano Ruiz J, Pavel A, et al. Frontal Fibrosing Alopecia shows robust Th1 and JAK3 skewing. Br J Dermatol. 2020. doi:10.1111/bjd.19040

35. Head J, Lawrence B. The aryl hydrocarbon receptor is a modulator of anti-viral immunity. Biochem Pharmacol. 2009;77(4):642-653. doi:10.1016/j.bcp.2008.10.031 
36. Giannone J, Li W, Probst M, Okey A. Prolonged depletion of AH receptor without alteration of receptor mRNA levels after treatment of cells in culture with 2,3,7,8-tetrachlorodibenzo-p-dioxin. Biochem Pharmacol. 1998;55(4):489-497. doi:10.1016/S0006-2952(97)00493-0

37. Doche I, Pagliari C, Hordinsky M, et al. Overexpression of the aryl hydrocarbon receptor in frontal fibrosing alopecia and lichen planopilaris: a potential pathogenic role for dioxins?: an investigational study of 38 patients. J Eur Acad Dermatol Venereol. 2020. doi: $10.1111 /$ jdv. 16287

38. Munger K, Zhang S, O'Reilly E, et al. Vitamin D intake and incidence of multiple sclerosis. Neurology. 2004;62(1):60-65. doi:10.1212/01. WNL.0000101723.79681.38
39. Martens P, Gysemans C, Verstuyf A. Mathieu a. Vitamin D's effect on immune function. Nutrients. 2020;12(5):1248. doi:10.3390/ nu12051248

40. Bonefeld-Jørgensen E, Long M, Hofmeister M, Vinggaard A. Endocrine-disrupting potential of bisphenol A, Bisphenol A Dimethacrylate, 4-n-Nonylphenol, and 4-n-Octylphenol in vitro: new data and a brief review. Environ Health Perspect. 2007;115 (Suppl 1):69-76. 8. doi:10.1289/ehp.9368

41. Tziotzios C, Petridis C, Dand N, et al. Genome-wide association study in frontal fibrosing alopecia identifies four susceptibility loci including HLA-B*07:02. Nat Commun.

\section{Publish your work in this journal}

Clinical, Cosmetic and Investigational Dermatology is an international, peer-reviewed, open access, online journal that focuses on the latest clinical and experimental research in all aspects of skin disease and cosmetic interventions. This journal is indexed on CAS.
The manuscript management system is completely online and includes a very quick and fair peer-review system, which is all easy to use. Visit http://www.dovepress.com/testimonials.php to read real quotes from published authors. 